Burkitt's lymphoma cell line BJAB-LexR that has been made naturally resistant to TRAIL through long-term culture in the presence of increasing concentrations of the drug Lexatumumab, an anti-DR5 antibody, as well as BJAB cells with Six 1 overexpression. Performing experiments in both these contexts would allow us to distinguish between resistance genes that arise spontaneously versus resistance genes that are a direct effect of Six 1 expression. We performed a genome-wide loss of function screen using the GeneNet lentiviral shRNA library containing 200000 shRNAs with sequencing tags, allowing for high throughput deep sequencing. Afte selection for a particular phenotype, in this case TRAIL resistance, the shRNAs that promote or prevent this phenotype can be deduced from the frequency in which the shRNA is present in the selected population versus the unselected population. A library was constructed using sequence tag specific primers and deep sequencing was performed using Illumina sequencing technology. Bioinformatic analysis yielded a list of putative resistance genes that are currently being validated in the BJAB system and also in MDAMB231 breast cancer cells that have been made resistan to TRAIL in a similar fashion. With this unbiased approach, we aim to identify not only known components of the TRAIL pathway that may be deregulated but also completely novel pathways of TRAIL resistance that could be targeted for improved cancer therapy.

101

POSTER

Smac Mimetic Induces Apoptosis and Synergizes with TRAIL in Inflammatory Breast Cancer Cells in an IAP-Dependent and TNF-a-Independent Mechanism

G.R. Devi ${ }^{1}$, J.L. Allensworth ${ }^{1}$, S.J. Sauer ${ }^{1}$, M.M. Morse ${ }^{2}$, H.K. Lyerly ${ }^{1}$. ${ }^{1}$ Duke University Medical Center, Surgery and Comprehensive Cancer Ctr, Durham NC, USA; ${ }^{2}$ Duke University Medical Center, Medicine and Comprehensive Cancer Ctr, Durham NC, USA

Background: Acquired therapeutic resistance due to tumor cell adaptation to persistent therapeutic stress is an unmet challenge in inflammatory breast cancer (IBC), designated as an orphan cancer with the worst survival rates amongst all breast cancers.

IBC tumor cells behave differently from other breast cancer by reprogramming the protein synthesis machinery enabling constitutive translation of key survival proteins. We have identified overexpression of $\mathrm{X}$-linked Inhibitor of Apoptosis Protein (XIAP), the most potent mammalian caspase inhibitor via post-transcriptional mechanisms as a dominant feature of anti-apoptotic dysregulation involved in acquired therapeutic resistance to epidermal growth factor receptor (EGFR, and/or ErbB2/HER2)-targeting drugs like trastuzumab and lapatinib as well as apoptosis inducing agents such as TRAIL (TNF-related apoptosis inducing ligand). The second mitochondria-derived activator of caspases (Smac) protein is a potent antagonist of IAP proteins including XIAP and the basis for the development of Smac mimetic drugs.

Methods: In the present study, we have characterized bivalent Smac mimetics as single agents and in combination with TRAIL to determine the ability of these agents to induce cell death in basal type (SUM149) and ErbB2-overexpressing (SUM190) IBC cell lines, as well as in an isogenic SUM149 derivative with stable XIAP overexpression (SUM149 wtXIAP) and an isogenic SUM190 derivative with stable XIAP knockdown (SUM190 shXIAP).

Results: Here, we report that Smac mimetic induces cell death as a single agent in TRAIL-insensitive SUM190 (ErbB2-overexpressing) cells via pan-IAP antagonism. In addition, Smac mimetic significantly increased potency of TRAIL-induced apoptosis in a TRAIL-sensitive SUM149 (triple negative, EGFR-activated) cells. SUM149 and SUM190 are established patient tumor-derived IBC models. Treatment with SMAC mimetic caused rapid clAP1/2 degradation, caspase activation, PARP cleavage, NF- $\mathrm{KB}$ activation. A modest increase in TNF- $\alpha$ production in SUM190 cells was observed. Exogenous TNF- $\alpha$ addition did not increase the efficacy of the Smac mimetic. Neutralizing antibodies against TNF- $\alpha$ or TNFR1 knockdown did not reverse cell death. However, pan-caspase inhibitor $\mathrm{Q}-\mathrm{VD}-\mathrm{OPh}$ reversed cell death.

Conclusion: By demonstrating that Smac protein primes IBC cells for death in an IAP-dependent manner, these findings support the development of Smac mimetics for IBC treatment.

\section{Cellular Therapies}

102 POSTER

Autoimmune Regulator (Aire) is a Transcriptional Link Between Autoimmunity and Thymus Cancer

E. Oliveira ${ }^{1}$, C. Macedo ${ }^{1}$, C. Collares ${ }^{1}$, E. Sakamoto-Hojo ${ }^{1}$, E. Donadi ${ }^{1}$ G. Passos ${ }^{1}$. ${ }^{1}$ Medical School of Ribeirao Preto-USP, Genetics, Ribeirao Preto - SP, Brazil

Background: The regulation of negative selection of thymocytes during the induction of self-tolerance in the thymus is dependent on genes expressed by medullary thymic epithelial cells (mTECs). In these cells, the transcription factor autoimmune regulator (Aire) regulates the expression of a large set of downstream genes; of these Aire-dependent genes, $45 \%$ encode peripheral tissue antigens (PTAs), which represent the diverse tissues and organs. Due to the diversity of genes controlled by Aire, this phenomenon then has been termed 'promiscuous gene expression' (PGE). In addition, evidence showed that thymomas (a tumor originated from thymic epithelial cells) express Aire at low levels compared to normal thymus. This suggests that Aire deficiency might have an oncogenic impact on the development of thymomas. In addition, thymomas patients can develop autoimmune diseases being myasthenia gravis (MG) the commonest. Accordingly, we raised the hypothesis that in addition to PTAs genes, Aire could controls genes associated to carcinogenesis.

Material and Methods: We initially used siRNA to knockdown Aire by in vivo electro-transfection of the thymus of BALB/c mice. Aire mRNA levels from purified medullary thymic epithelial cells (mTECs) was then accessed by qRT-PCR and Aire protein by immunofluorescence microscopy.

Results: Aire-siRNA reached medullary thymic epithelial cells (mTECs) down-regulating Aire mRNA and Aire protein levels. To explore the effect of Aire knockdown over the transcriptome, we used the whole functional genome Agilent microarray method allowing determining the modulation of a variety of PTA genes as well as the cancer associated p53 and Vegfa and Vegfb genes. After Aire knockdown, p53 gene was down-regulated while the two Vegf family members were up-regulated. Noteworthy, down-regulation of p53 favors tumorigenesis and up-regulation of Vegf favors angiogenesis. Two essential processes for cancer progression in the thymus. Moreover, PTA genes AchR, Títin and RyR, which represent muscle autoantigens, and therefore associated to immunologic autoreactivity found in MG were up-regulated.

Conclusions: These results show that expression of Aire is a link between cancer and autoimmunity and might represent a key molecular target in controlling these diseases in thymus.

Financial support: FAPESP, CNPq and CAPES (Brazil).

103 POSTER

Generation and Characterisation of Neratinib-resistant Breast Cancer Cell Line Variants

S. Breslin ${ }^{1}$, S. Rani ${ }^{1}$, C. Corcoran ${ }^{1}$, K. O'Brien ${ }^{1}$, L. O'Driscoll ${ }^{1} .{ }^{1}$ Trinity College Dublin, School of Pharmacy and Pharmaceutical Sciences \& Trinity Biomedical Sciences Institute, Dublin 2, Ireland

Background: HER2 overexpression occurs in $20-25 \%$ of breast cancer cases and is commonly treated with regimes including HER2-targeting trastuzumab and/or lapatinib. Neratinib is an irreversible EGFR, HER2 and HER4 tyrosine kinase inhibitor currently in phase III clinical trials for breast cancer treatment. Not all HER2-positive breast cancer patients respond to HER2-targeted drugs and some, who initially respond, subsequently relapse. We aimed to investigate the potential of cells to acquire neratinib resistance and investigate the resulting associated effects of resistance on cell behaviour

Materials and Methods: Neratinib-resistant variants of breast cancer cell lines SKBR3 and HCC1954 were developed by continuous exposure to neratinib over $\sim 4$ months. These variants were characterised and compared with controls in terms of resistance/cross-resistance to HER2-targeting drugs, proliferation, motility, invasion and anoikis. Growth of these variants in traditional 2D culture was compared with a forced-floating method of $3 D$ culture using poly-HEMA coated dishes.

Results: Neratinib-resistant variants were successfully established for both SKBR3 and HCC1954 cell lines. Specifically, HCC1954 resistant $\left(\mathrm{HCC} 1954-\mathrm{N}^{\mathrm{R}}\right)$ cells are $6.6 \pm 0.36$ fold more resistant than their control population (HCC1954-N ${ }^{S}$ ). SKBR3 resistant cells $\left(S K B R 3-N^{R}\right)$ are at least 20 fold more resistant to neratinib than their control (SKBR3-N ${ }^{S}$ ). HCC 1954- $N^{R}$ cells were found to be cross-resistant to afatinib and lapatinib at $37 \pm 7.23$ fold and $10 \pm 0.8$ fold, respectively. $\mathrm{HCC} 1954-\mathrm{N}^{\mathrm{S}}$ are innately resistant to trastuzumab; HCC1954- $\mathrm{N}^{\mathrm{R}}$ cells maintain this resistance. HCC1954- $\mathrm{N}^{R}$ and HCC1954- $\mathrm{N}^{S}$ cells proliferate at significantly $(p=0.011)$ different rates. Wound-healing assays indicated that motility of HCC1954$\mathrm{N}^{\mathrm{R}}$ cells is not significantly $(\mathrm{p}=0.117)$ different to that of HCC1954- $\mathrm{N}^{S}$ 
cells. However, HCC1954- $\mathrm{N}^{R}$ are more $(p=0.001)$ invasive and resistant to anoikis $(p=0.003)$ than HCC1954- $\mathrm{N}^{\mathrm{S}}$. Furthermore, Western blot analysis of HCC1954- $\mathrm{N}^{R}$ and HCC1954- $\mathrm{N}^{S}$ grown in $2 \mathrm{D}$ and 3D culture showed higher HER2 expression and lower EGFR expression in $3 D$ versus $2 D$ samples of both the sensitive and resistant variants. Preliminary data indicates that the $\mathrm{IC}_{50}$ of neratinib in both HCC1954- $\mathrm{N}^{\mathrm{R}}$ and HCC1954$\mathrm{N}^{S}$ substantially increases when grown in $3 \mathrm{D}$, compared to $2 \mathrm{D}$ culture. Conclusion: Like trastuzumab and lapatinib, resistance to neratinib develops with time and cross-resistance to other HER2-targeting drugs occurs. Furthermore, acquired neratinib-resistance is associated with altered proliferation, invasion and anoikis. Growing cells in 3D alters expression of neratinib targets HER2 and EGFR, and so may be contributing to the altered response of 3D cultures to neratinib. Acknowledgements: Marie Keating Foundation PhD Scholarship at Trinity College Dublin, Trinity Foundation \& Science Foundation Ireland (08/SRC/B1410).

\section{4} POSTER MAGE-A3 Counteracts Cell Survival in Human Bladder Cancer Cell Lines

J. Hänze ${ }^{1}$, G. Zhou ${ }^{1}$, M. Henrici ${ }^{1}$, A. Hegele ${ }^{1}$, R. Blaheta ${ }^{2}$, R. Hofmann ${ }^{1}$, P. Olbert ${ }^{1} .{ }^{1}$ Philipps University, Urology and Pediatric Urology, Marburg, Germany; ${ }^{2}$ University, Urology and Pediatric Urology, Frankfurt, Germany

Background: The type I Melanoma Antigen Genes, such as MAGE-A3 are expressed in various cancers including bladder cancer the second most prevalent urogenital cancer. In normal tissues, MAGE-A3 is restricted to testis and placenta representing tissues with low antigen presentation. Thus, MAGE-A3 is under investigation as possible target for cance immunotherapy. Furthermore, MAGE-A3 may exert oncogenic functions as demonstrated in myeloma cells. This study aimed to characterize possible functions of MAGE-A3 with respect to cell survival in bladder cancer cells. Methods: Several human bladder cell lines (UMUC, 5637, T24, EJ28, BFTC, TCC-SUP, HU456, HT) were analyzed for MAGE-A3 mRNA expression by quantitative realtime RT-PCR. Functional studies of MAGEA3 related to cell survival as measured by colony formation and XTT assay were performed. For this purpose, silencing of MAGE-A3 by RNAinterference employing synthetic siRNA was established in these cell lines. Results: MAGE-A3 mRNA levels where highest in UMUC-3, 5637 and T24 cells whereas no detectable levels were observed in EJ28 cells. BFTC, TCC-SUP, HU456, HT cells exhibited intermediate MAGE-A3 mRNA levels. In the next step, potent siRNA silencing of MAGE-A3 mRNA was established in UMUC-3, 5637 and T24 cells and approximately 10 -fold downregulation of MAGE-A3 could be proven. EJ28 cells that displayed no detectable MAGE-A3 expression served as control cells in the following functional studies. Interestingly, silencing of MAGE-A3 resulted in significant increased cell survival as demonstrated by clonogenic survival and XTT assay in all three MAGE-A3 expressing cells, whereas no effect was observed in EJ28 cells.

Conclusions: Our data demonstrated an inhibitory effect of MAGE-A3 on cell survival in bladder cancer cell lines as demonstrated by enhanced cell survival after potent downregulation of MAGE-A3 by RNA interference. The specificity of these unexpected observations is supported by the non-MAGE-A3 expressing EJ28 cell line that did not alter cell survival after MAGE-A3 siRNA treatment. Ongoing studies will focus on signal transduction pathways mediated by MAGE-A3 regarding cell survival. Overall, this study explored an important so far non-described anti-survival function of MAGE-A3 in several bladder cancer cells.

105

POSTER

\section{Functional Profiling of Clear Cell Ovarian Cancer}

R. Miller ${ }^{1}$, R. Brough ${ }^{1}$, I. Bajrami ${ }^{1}$, S. Kaye ${ }^{1}$, S. Banerjee ${ }^{1}$, C. Lord ${ }^{1}$, A. Ashworth ${ }^{1} .{ }^{1}$ Institute of Cancer Research, London, United Kingdom

Ovarian clear cell carcinoma (OCCC) is characterised by low response rates to standard chemotherapy and poorer prognosis than the more common serous ovarian cancer subtype. Recently ARID1A loss of function mutations have been identified in up to $50 \%$ of OCCC. ARID1A is a component of the SWI/SNF chromatin-remodeling complex, which regulates many cellular processes including differentiation, proliferation and DNA repair. ARID1A functions as a tumour suppressor and the high incidence of this mutation make it an attractive therapeutic target for OCCC. Based on our current understanding of ARID1A function it is difficult to predict a therapeutic strategy for these tumours. Therefore we adopted a relatively unbiased approach using integrated functional and molecular profiling of ARID1A mutant and wild-type tumour cell models.

The results generated from functional analysis using high-throughput screening with chemical and siRNA libraries have identified critical genetic dependencies and potential therapeutics in a series of clear cell ovarian cancer models. A small molecule tyrosine kinase inhibitor has been established as being selective for ARID1A mutant tumour cell lines. In vivo and in vitro work work is underway to determine the mechanism underlying this selectivity.

The results to date provide a framework for the discovery of therapeutic targets for clear cell ovarian cancer characterised by ARID1A mutations, using an integrated approach. Re-validation of these preliminary results is ongoing in order to characterise new genetic dependencies for this disease.

106 POSTER Identifying KRAS New Synthetic Lethality Relationships in Cancer

S. Carvalho ${ }^{1}$, M. Aarts ${ }^{1}$, J. Riffell ${ }^{1}$, C. Torrance ${ }^{2}$, C.J. Lord ${ }^{1}$, A. Ashworth ${ }^{1}$ ${ }^{1}$ Institute of Cancer Research, Gene Function, London, United Kingdom; ${ }^{2}$ Horizon, Horizon Discovery Building, Cambridge, United Kingdom

Background: KRAS is among the most commonly mutated human cancer genes, and is mutated in approximately $20 \%$ of human tumours. In colorectal cancer KRAS mutation is a predictor of anti-EGFR treatment resistance and different KRAS mutations can lead to different anti-EGFR treatment responses. Despite this, KRAS has proven difficult to target with small molecule inhibitors. Identifying synthetic lethal interactions that occur only in the context of tumour specific KRAS mutations represents one approach that could be used to target tumour cells.

Material and Methods: To identify KRAS synthetic lethal interactions we are generating functional profiles of constitutively activated KRAS mutan cancers, using an integrated approach that involves cell viability screening. On a panel of isogenic tumour cell lines carrying different KRAS mutations (G12D, G12S, G12V and G13D) we performed siRNA screens using a library targeting kinases, kinase-related and tumour suppressor genes. In addition, by using drug sensitivity screening using a library of commonly used oncology agents including a large number of in-development targeted agents, we have identified KRAS selective agents. By assessing the effect on cell viability of each siRNA/ drug in each cell model we were able to identify KRAS mutation specific effects.

Results: By using high throughput functional viability profiling in KRAS mutant models we have been able to identify a series of genetic dependencies specific for tumour cells with oncogenic KRAS mutations. We have also preliminary results with small molecule inhibitors, which target some of the genes that we identified as being critical for the survival of KRAS mutant cancers.

Conclusions: Using functional viability profiling of a series of KRAS isogenic cell lines we have now identified several KRAS synthetically lethal candidate genes. Our subsequent work will focus on assessing the generality of these observations as well as dissection of the molecular mechanisms responsible for these KRAS mutant selective effects.

107

Multi-level Gene Expression Regulation Effects of the NAMPT Inhibitor FK866 in a Model of Acute T Cell Leukemia

C. Zucal $^{1}$, V. D'Agostino ${ }^{1}$, T. Tebaldi ${ }^{1}$, G. Sociali ${ }^{2}$, S. Bruzzone ${ }^{2}$, A. Quattrone ${ }^{1}$, A. Nencioni ${ }^{3}$, A. Provenzani ${ }^{1} .{ }^{1}$ Centre for Integrative Biology CIBIO, University of Trento, Trento, Italy; ${ }^{2}$ Department of Experimental Medicine-Section of Biochemistry, University of Genoa, Genoa, Italy;

${ }^{3}$ Department of Internal Medicine, University of Genoa, Genoa, Italy

Background: Nampt/PBEF/visfatin gene encodes for a key enzyme in the regulation of NAD biosynthesis from the natural precursor nicotinamide. Its inhibition, by the low molecular weight compound FK866 (also known as APO866), has an enormous impact on the maintenance of cell viability because of the fundamental functions of the NAD molecule in cell physiology. Tumor cells are more vulnerable to inhibition of NAD synthesis than non-tumor cells; particularly, $T$ cell leukemia cell lines selectively undergo apoptosis when endogenous NAD synthesis is limited using FK866.

Material and Methods: We applied FACS analysis to evaluate the effect of FK866 in Jurkat cell line. The efficacy of the drug was also confirmed by assessing the intracellular NAD-levels through an enzyme cycling assay. To investigate if FK866 induced gene expression changes in leukemic T cells, RNA samples extracted from treated or control cells were used for gene expression microarray analysis. RNA was derived from standard whole cell lysates, cytoplasmic lysates and sucrose gradient fractionation (sub-polysomes, polysomes). A differential gene expression analysis of the subsets was performed along with a polysomal/subpolysomal distribution in treated or untreated cells and Gene Ontology category overrepresentation. Results: Dose-response curves revealed a decrease in the number of cycling cells following treatment with FK866 and the calculated $\mathrm{EC}_{50}$ at 48 hours was $5.5 \mathrm{nM}$. We treated Jurkat cells for 48 hours at the chosen 